\title{
Strangeness in the Proton Wave Function?
}

\author{
P. M. GENSINI
}

Dipartimento di Fisica dell'Università - I-06100 Perugia

INFN - Sezione di Perugia

(Nzovo Cimento A, 102, 75 (1989))

The published version of this paper contains quite a number of misprints (all but two absent in the original manuscript), none of which has any influence on the conclusions drawn therein, but which nonetheless make the paper itself much less clear than intended by the author.

On p. 76: in line 4, read $«\left(q^{2}=0\right) »$ instead of $«\left(q_{2}=0\right) »$;

on p. 77: in the line above eq. (12), read «SU $3 U_{3} \times S U_{3} »$ instead of $« S U_{2} \times S U_{3} »$;

on p. 78: in line 11 , read «(35 \pm 5$) \mathrm{MeV} »$ instead of « $(35 \div 5) \mathrm{MeV}$; in line 13 , read «628 MeV» instead of « $638 \mathrm{MeV}$;

on p. 81: the first term in eq. (14) should be $« \Sigma^{-}-\Sigma^{+} »$ instead of $« \Sigma^{-}+\Sigma^{+} »$; in the second term of eq. (18), read «( $\left(3 f^{2}+4 f d-d^{2}\right) »$ instead of $"\left(3 f^{2}-4 f d-d^{2}\right) »$;

on p. 82: in the first line of the second paragraph, read « $\mu_{0}^{2} \cong 0$ » instead of $« \mu_{0}^{2}$," and, in the fifth line of the same, "meson-meson" instead of "mesonmeson"; in the line above eq. (28), read "SU(3)-violating» instead of "SU(3)-violation» (this error was also in the manuscript version);

on p. 83: in the first line of the second paragraph, there shoud be a comma before «eqs. (29)-(30)" (towards the end of the line); in the fifth line of the same, read «OLCPT» instead of «OLPCPT»; the second term in eq. (30) should have a minus sign in front of it; two lines above eq. (33), the r... s. of the inequality should read «15.33 $33_{-2.71 »}^{+4.09}$ instead of $« 1533_{-2.71}^{+4.09} »$;

on p. 84: in the seventh line of the third paragraph, remove the first comma (which, alas, was there in the manuscript as well!); 
on p. 85: in the line above eq. (38), read «I» instead of «l»;

on p. 86: in the third line of the second paragraph, read "the use of a" instead of «the use a»;

on p. 87: in line 6, read "thoroughly" instead of "throughly»;

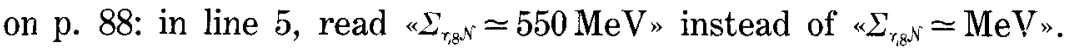

Both the author and the editors apologize for the inconveniences, according to their own shares of errors! 